\title{
Microbiological profile and treatment outcome of emphysematous pyelonephritis: experience from two teaching hospitals of Bangladesh
}

\author{
Chowdhury Ja, Biswas NK ${ }^{a}$, Kanta $S^{b}$, Rahim MAc, Haque WMMc, Iqbal $\mathbf{S}^{d}$, \\ Uddin KNe
}

\begin{abstract}
Background: Emphysematous pyelonephritis (EPN) is a rare severe necrotizing infection of the renal parenchyma, collecting system and/or perirenal tissues. Patients with diabetes mellitus, renal stones and renal tract obstruction are at risk for development of EPN. Escherichia coli is the most common aetiological agent and treatment is aimed at infection control and interventions in appropriate cases. This study aimed to identify the aetiological agents responsible for EPN and the outcome of such cases.
\end{abstract}

Methods: This observational study was done in BIRDEM General Hospital and Bangabandhu Sheikh Mujib Medical University over a period of three years (2011-2013). Twenty adult EPN cases were included in this study.

Results: The mean age of the study participants was 59.2 years with female predominance (80\%). Risk factors included diabetes mellitus (90\%), renal stones (5\%) and kidney transplant recipient (5\%). Fever, loin pain and dysuria were common. Six patients had class 1 EPN, seven had class 2 disease, five had class 3 and two patients had class 4 EPN. Aetiological agents were cultured in 19 cases; E. coli in 12 (68\%), Klebsiella in three (16\%), Pseudomonus in two (11\%) and Proteus and Enterococcus, one each (5\% each). Carbapenems appeared as the most sensitive antibiotic. Along with antibiotic, half of the patients required interventions; percutaneous drainage in two, open drainage in two and six patients required nephrectomy. Regarding outcome, five patients died, 10 patients recovered completely and five patients recovered with residual renal dysfunction.

Conclusion: E. coli was the most common causative agent in present EPN cohort. Outcome was poor. With improved management strategy, combining nephrologists, intervention radiologists and urologists - all together, a good outcome is expected.

Key words: aetiological agents, emphysematous pyelonephritis, outcome.

(BIRDEM Med J 2021; 11(2): 108-111)

\section{INTRODUCTION}

Emphysematous pyelonephritis (EPN) is a severe necrotizing infection of the renal parenchyma. It causes

\section{Author information}

a. Juli Chowdhury, Nirmol Kumar Biswas, Junior Consultant, Medicine, National Institute of Cardiovascular Diseases (NICVD), Dhaka, Bangladesh.

b. Shamima Sharmin Kanta, Senior Medical Officer, Respiratory Medicine, BIRDEM General Hospital, Dhaka, Bangladesh.

c. Muhammad Abdur Rahim, Wasim Md. Mohosin Ul Haque, Associate Professor, Nephrology, BIRDEM General Hospital, Dhaka, Bangladesh.

d. Sarwar Iqbal, Professor, Nephrology, BIRDEM General Hospital, Dhaka, Bangladesh.

e. Khawja Nazim Uddin, Professor, Internal Medicine, BIRDEM, General Hospital, Dhaka, Bangladesh.

Address of correspondence: Juli Chowdhury, Junior Consultant, Medicine, NICVD, Dhaka, Bangladesh. Email: chowdhuryjuliyash2013@gmail.com

Received: September 26, 2020

Revision received: January 27, 2021

Accepted: February 28, 2021 gas formation within the collecting system, renal parenchyma and/or perirenal tissues. ${ }^{1}$ Gas in the renal pelvis alone, without parenchymal gas, is often referred to as emphysematous pyelitis. ${ }^{2}$ EPN is common in persons with diabetes and the presentation of EPN is similar to that of acute pyelonephritis. ${ }^{3}$ Renal stones are another predisposing condition and transplanted kidneys may be susceptible to EPN because of associated high-risk factors in the recipient such as diabetes and immunosuppression. ${ }^{1,3,4}$ This infection often has a fulminating course and can be fatal if left untreated.

The mortality rate associated with the condition was high before the advent of antibiotics. However, advances in imaging technology, control of diabetes, resuscitative management and minimally invasive treatment have improved the outcome in patients with EPN. Although nephrectomy may be the quickest way of treating the infection source, renal function is compromised in many patients. Therefore, a strategy to save nephrons may 
be very desirable. ${ }^{5}$ Effective conservative therapy includes early initiation of effective antibiotics.

Escherichia coli is the most common organism responsible for EPN ${ }^{1,3,6}$ and other form of urinary tract infections. Other organisms include Klebsiella, Pseudomonas, Candida and rarely gas forming organisms. ${ }^{1}$ This study was designed to evaluate microbiological agents responsible for EPN and outcome of such patients.

\section{METHODS}

This observational study was carried out in Bangladesh Institute of Research and Rehabilitation in Diabetes, Endocrine and Metabolic Disorders (BIRDEM) General Hospital, Dhaka, Bangladesh and Bangabandhu Sheikh Mujib Medical University (BSMMU), Dhaka, Bangladesh from January 2011 to December 2013. Total 20 adult (age $>18$ years) patients with a diagnosis of EPN were consecutively included in this study. Patients' clinical, microbiological and outcome data were recorded in case record forms. The study protocol was approved by the Bangladesh College of Physicians and Surgeons (BCPS).

\section{RESULTS}

Total patients were 20 (females $16,80 \%$ ) with a mean age of $59.2 \pm 16.7$ (range $38-77$ ) years. Eighteen $(90 \%)$ patients were diabetic with poor diabetic control (HbA1c $>7 \%$ in 16), two (10\%) patients had chronic kidney disease, one (5\%) patient had renal stone and one $(5 \%)$ patient was a kidney transplant recipient. Fever was the most common presentation and others are shown in Table I. Neutrophil leukocytosis and raised inflammatory markers were common (Table II). E. coli was the most common organism identified on urine culture (Table III) and carbapenems appeared as the most sensitive antibiotic (Table IV). Left kidney was involved in 14 cases, right kidney in four, bilateral in one and transplanted kidney was involved in one case. Six patients had class $1 \mathrm{EPN}$, seven had class 2 disease, five had class 3 and two patients had class 4 EPN. ${ }^{1}$ All patients required resuscitative measures, insulin for diabetics and other supportive measures; antibiotic alone in $10(50 \%)$ cases, antibiotics and percutaneous drainage in two $(10 \%)$, open drainage in two $(10 \%)$ and nephrectomy in six $(30 \%)$ cases. Half of the patients recovered completely, one-fourth had incomplete recovery and one-fourth died (Table $\mathrm{V}$ ).
Table I Clinical presentation of patients with emphysematous pyelonephritis $(\mathrm{N}=20)$

\begin{tabular}{lcc}
\hline Clinical feature & Frequency & Percentage \\
\hline Fever & 20 & 100 \\
Dysuria & 17 & 85 \\
Loin pain & 15 & 75 \\
Nausea and/or vomiting & 13 & 65 \\
Shock & 8 & 40 \\
Altered consciousness & 7 & 35 \\
Oliguria & 6 & 30 \\
\hline
\end{tabular}

Table II Abnormal laboratory test of patients with emphysematous pyelonephritis $(\mathrm{N}=20)$

\begin{tabular}{lcc}
\hline Investigation & Frequency & Percentage \\
\hline Low haemoglobin & 14 & 70 \\
Leukocytosis & 13 & 65 \\
Thrombocytopaenia & 4 & 20 \\
Raised erythrocyte & 20 & 100 \\
sedimentation rate & & \\
Raised C-reactive protein & 20 & 100 \\
Pyuria & 18 & 90 \\
Haematuria & 3 & 15 \\
Acute kidney injury & 12 & 60 \\
Altered liver biochemistry & 7 & 35 \\
Abnormal urine culture & 19 & 95 \\
Abnormal blood culture & 11 & 55 \\
\hline
\end{tabular}

Table III Isolated bacterial pathogens from urine culture $(\mathrm{N}=19)$

\begin{tabular}{lcc}
\hline Bacteria & Frequency & Percentage \\
\hline E. coli & 12 & 63.2 \\
Klebsiella & 3 & 15.8 \\
Pseudomona & 2 & 10.5 \\
Proteus & 1 & 5.3 \\
Enterococcus & 1 & 5.3 \\
\hline
\end{tabular}




$\begin{aligned} & \text { Table IV Antibiotic sensitivity pattern of isolated } \\
& \text { organisms from urine }(\mathrm{N}=19)\end{aligned}$
\begin{tabular}{lcc}
\hline Name of antibiotic & Sensitive & Percentage \\
\hline Imipenem & 15 & 78.9 \\
Meropenem & 15 & 78.9 \\
Piperacillin-tazobactum & 13 & 68.4 \\
Colistin & 12 & 63.2 \\
Ceftazidim & 8 & 42.1 \\
Ceftriaxone & 3 & 15.8 \\
Linezolide & 5 & 26.3 \\
Amikacin & 5 & 26.3 \\
Gentamicin & 5 & 26.3 \\
Netilmycin & 6 & 31.6 \\
\hline
\end{tabular}

Table V Treatment and outcome of patients with emphysematous pyelonephritis $(\mathrm{N}=20)$

\begin{tabular}{lcc}
\hline Treatment given & Frequency & Percentage \\
\hline Antibiotic only & 10 & 50 \\
Antibiotic and percutaneous & 2 & 10 \\
drainage & & \\
Antibiotic and open drainage & 2 & 10 \\
Nephrectomy & 6 & 30 \\
Outcome & & \\
Complete recovery & 10 & 50 \\
Recovered with residual renal & 5 & 25 \\
dysfunction & & \\
Death & 5 & 25 \\
\hline
\end{tabular}

\section{DISSCUSSION}

EPN is an uncommon diagnosis. Most published series consist of small numbers of cases. We reported a series of 20 cases over a three-year period. Mean age of our patients was comparable with those reported by Huang and Tseng but older than those reported by Samad T et al and Khan MMR et al from Bangladesh. ${ }^{1,3,6}$ In all these series, there was female predominance of cases. Diabetes was the most common risk factor in all these series accounting for $90-100 \%$ cases.

E. coli is the most common aetiological agent for urinary tract infection; EPN is not an exception. In our series, 12 out of 20 cases were due to E. coli and three cases were due to Klebsiella. In a recent report from Bangladesh, $E$. coli was the most common identified agent, responsible in $70 \%$ cases of EPN. ${ }^{6}$ Extended-spectrum beta-lactamase producing $E$. coli are also reported as causative agents for EPN from Bangladesh. ${ }^{7}$ Overall $E$. coli and Klebsiella constitute over $90 \%$ of all EPN cases reported from India ${ }^{8,9}$, Pakistan $^{10}$ and other countries. ${ }^{5,11}$ Gas in EPN may be identified by X-ray, ultrasonography and CT scan. There is different classification system for EPN; but computed tomography (CT) classification by Huang and Tseng ${ }^{1}$ is the most commonly used one, as in our series. In our study, class $2 \mathrm{EPN}$ was most common, followed by class 1 and 3 and class 4 was the least common. In previous two series from Bangladesh, class 2 EPN was the most common and class 4 was the lest common $^{3,6}$ but class 4 EPN with concomitant emphysematous cystitis is reported very recently from Bangladesh. $^{7}$

Management strategy for EPN includes fluid resuscitation, intravenous antibiotics, insulin in diabetics and surgery/intervention in selected cases. Huang and Tseng proposed management strategies depending up on $\mathrm{CT}$ class and risk factors (shock, altered sensorium, thrombocytopaenia and renal failure) but now-a-days more conservative approaches are practised with favourable outcomes. ${ }^{1,5,7}$

Outcome of EPN has improved over past decades, mostly resulting from broad spectrum antibiotics and minimally invasive techniques. In our series, mortality was high (25\%), while Samad T et al and Khan MMR reported a low mortality rate (6-7\%) from Bangladesh. ${ }^{3,6}$ Irfan AM et al reported no mortality in a series of 20 cases from Pakistan. ${ }^{10}$

One-fourth of our cases improved with residual renal function impairment. We could not follow up these cases prospectively; a follow up renogram could identify split function of kidneys. A prospective, multi-center study may be done in future.

In conclusion, $E$. coli was the most common causative agent in the present study followed by Klebsiella. CT scan and ultrasonography (USG) are the main tools to diagnose the disease but USG can miss the diagnosis. So, CT scan should be done in all suspected cases of EPN. Overall, outcome was poor with $25 \%$ mortality. With an improved management strategy by combining nephrologists, intervention radiologists and urologists - all together, a good outcome is expected.

Authors' contribution: JC planned the study, collected data and drafted manuscript. NKB, SSK collected data. 
MAR searched literature, critically reviewed and revised the manuscript. KNU was the overall supervisor. All authors read and approved the final manuscript for submission.

Conflicts of interest: Nothing to declare.

\section{REFERENCES}

1. Huang JJ, Tseng CC. Emphysematous pyelonephritis: Clinicoradiological classification, management, prognosis, and pathogenesis. Arch Intern Med 2000; 160: 797-805.

2. Hiremath R, Mahesh, Padala KP, Swamy K, Pailoor A. A Rare Case of Pneumoureter: Emphysematous Pyelitis versus Emphysematous Pyelonephritis. J Clin Diagn Res 2015 Nov; 9(11): TD03-TD05.

3. Samad T, Haque WMM, Iqbal S. Emphysematous Pyelonephritis: Experience of Managing 15 Consecutive Cases in a Tertiary Care Hospital of Bangladesh. BIRDEM Med J 2018; 8(3): 246-50.

4. Oliveira CC, Garcia PD, Vieroa RM. Emphysematous pyelonephritis in a transplanted kidney. Autops Case Rep 2016 Oct-Dec; 6(4): 41-47.

5. Karthikeyan VS, Manohar CMS, Mallya A, Keshavamurthy R, Kamath AJ. Clinical profile and successful outcomes of conservative and minimally invasive treatment of emphysematous pyelonephritis. Cent European J Urol 2018; 71: 228-233.
6. Khan MMR, Rahman F, Rahman S, Chowdhury ATMM, Hasan MM. Management of emphysematous pyelonephritis and subsequent renal functional outcome. Ibrahim Card Med J 2017; 7(1 \& 2):57-63.

7. Rahim MA, Jahan I, Chowdhury TA, Ananna MA, Iqbal S. Class 4 emphysematous pyelonephritis with emphysematous cystitis: report of a rare case from Bangladesh. Tropical Doctor. DOI: 10.1177/0049475520983641

8. Misgar RA, Mubarik I, Wani AI, Bashir MI, Ramzan M, Laway BA. Emphysematous pyelonephritis: A 10-year experience with 26 cases. Indian J Endocrinol Metab 2016 Jul-Aug; 20(4): 475-480.

9. Eswarappa M, Suryadevara S, John MM, Kumar M, Reddy SB, Suhail M. Emphysematous Pyelonephritis Case Series From South India. Kidney International Reports 2018; 3 : 950-955.

10. Irfaan AM, Shaikh NA, Jamshaid A, Qureshi AH. Emphysematous Pyelonephritis: A single center review. Pak J Med Sci 2020 Jan; 36(1) Special Suppln. ICON 2020; S83- S86.

11. Sokhal AK, Kumar M, Purkait B, Jhanwar A, Singh K, Bansal A, et al. Emphysematous pyelonephritis: Changing trend of clinical spectrum, pathogenesis, management and outcome. Turk J Urol 2017 Jun; 43(2): 202-209. 